University of Nebraska - Lincoln

DigitalCommons@University of Nebraska - Lincoln

Faculty Publications: Materials Research

Science and Engineering Center

Materials Research Science and Engineering

Center

7-12-2004

\title{
Field-controlled domain-wall resistance in magnetic nanojunctions
}

John D. Burton

University of Nebraska-Lincoln, jburton2@unl.edu

Arti Kashyap

University of Nebraska-Lincoln, akashyap@Inmiit.ac.in

Mikhail Ye. Zhuravlev

University of Nebraska-Lincoln, myezhur@gmail.com

Ralph Skomski

University of Nebraska-Lincoln, rskomski2@unl.edu

Evgeny Y. Tsymbal

University of Nebraska-Lincoln, tsymbal@unl.edu

See next page for additional authors

Follow this and additional works at: https://digitalcommons.unl.edu/mrsecfacpubs

Part of the Materials Science and Engineering Commons

Burton, John D.; Kashyap, Arti; Zhuravlev, Mikhail Ye.; Skomski, Ralph; Tsymbal, Evgeny Y.; Jaswal, Sitaram; Mryasov, O.N.; and Chantrell, R.W., "Field-controlled domain-wall resistance in magnetic nanojunctions" (2004). Faculty Publications: Materials Research Science and Engineering Center. 34.

https://digitalcommons.unl.edu/mrsecfacpubs/34

This Article is brought to you for free and open access by the Materials Research Science and Engineering Center at DigitalCommons@University of Nebraska - Lincoln. It has been accepted for inclusion in Faculty Publications:

Materials Research Science and Engineering Center by an authorized administrator of DigitalCommons@University of Nebraska - Lincoln. 


\section{Authors}

John D. Burton, Arti Kashyap, Mikhail Ye. Zhuravlev, Ralph Skomski, Evgeny Y. Tsymbal, Sitaram Jaswal, O.N. Mryasov, and R.W. Chantrell 


\title{
Field-controlled domain-wall resistance in magnetic nanojunctions
}

\author{
J. D. Burton, A. Kashyap, M. Ye. Zhuravlev, R. Skomski, E. Y. Tsymbal, ${ }^{\text {a) }}$ and \\ S. S. Jaswal \\ Department of Physics and Astronomy, Center for Materials Research and Analysis, \\ University of Nebraska, Lincoln, Nebraska 68588
}

O. N. Mryasov and R. W. Chantrell

Seagate Research, Pittsburgh, Pennsylvania 15222

(Received 10 March 2004; accepted 12 May 2004)

\begin{abstract}
The electrical resistance of a constrained domain wall in a nanojunction is investigated using micromagnetic modeling and ballistic conductance calculations. The nanojunction represents two ferromagnetic electrodes connected by a ferromagnetic wire of $10 \mathrm{~nm}$ in length and of a few nanometers in cross section. We find that the anisotropy of the electrodes favors a localization of the domain wall within the constriction (wire) revealing a positive domain-wall resistance. An applied magnetic field moves the domain wall toward one of the electrodes and reduces its width. This compression of the domain wall leads to a sizeable enhancement of the domain-wall resistance. () 2004 American Institute of Physics. [DOI: 10.1063/1.1771455]
\end{abstract}

For decades the electrical resistance of a magnetic domain wall (DW) in metallic ferromagnets has been attracting considerable interest. ${ }^{1}$ Although it was predicted that DWs do not appreciably affect the resistance of bulk ferromagnets, ${ }^{2}$ recent advances in nanotechnology made it possible to measure a contribution to the resistance from a single DW. ${ }^{3-6}$ Interestingly, the DW resistance turned out in some cases to be negative, ${ }^{3}$ whereas in other cases to be positive. $^{4-6}$ Both results have found theoretical justifications. ${ }^{7-9}$ Experiments performed on Ni break junctions showed unexpectedly large magnetoresistance (MR) values that were attributed to a constrained DW. ${ }^{10}$ Although the results of these experiments are currently under debate, they stimulated theoretical studies of spin-dependent transport in constrained geometries using both free-electron models ${ }^{11-15}$ and ab initio calculations. ${ }^{16-18}$ In particular, a very abrupt DW was predicted for atomic-size junctions with the characteristic width of a few interatomic distances, which might explain the enhanced DW resistance. ${ }^{11}$ The interest to the electronic transport through DWs is additionally stimulated by possible applications of the MR associated with DWs in magnetoelectronic devices. From the point of view of applications, these devices need to demonstrate reproducible MR characteristics. For this purpose, electron lithography or focused ion beam techniques might be attractive and could provide typical dimensions of the nanostructures down to about $10 \mathrm{~nm}$.

In this study, we investigated the electrical resistance of a constrained DW in a nanojunction using micromagnetic modeling and ballistic conductance calculations. The nanojunction represents two ferromagnetic electrodes connected by a $10 \mathrm{~nm}$ long ferromagnetic wire of a few nanometers in cross section. We find that for electrodes with significantly different magnetic anisotropy, the DW can be trapped by the nanoconstriction. This results in a reduced conductance of the nanojunction, which implies a positive DW resistance. We show that the DW width and, therefore, the conductance can be controlled by an applied magnetic field. Due to high

${ }^{a)}$ Electronic mail: tsymbal@unl.edu anisotropy of an electrode, the increasing magnetic field compresses the DW and, therefore, enhances the DW resistance.

We consider a nanowire (constriction) made of soft magnetic material, such as $\mathrm{Ni}$, connecting two ferromagnetic electrodes that can be either both soft or one is soft and the other is hard. The constriction region is taken to be $10 \mathrm{~nm}$ $\times 2 \mathrm{~nm} \times 2 \mathrm{~nm}$. The micromagnetic modeling is performed using the Object Oriented MicroMagnetic Framework (OOMMF) code developed by NIST, which is based on solving the Landau-Lifshitz-Gilbert equation for magnetization. ${ }^{19}$ Each computational cell in the simulation is taken to be $1 \mathrm{~nm} \times 2 \mathrm{~nm} \times 2 \mathrm{~nm}$. This choice of cell size divides the constriction into ten layers of $1 \mathrm{~nm}$ thickness each.

For a given value of an applied magnetic field, the resulting micromagnetic configuration is used to calculate the conductance of the nanojunction. We assume that the transport is ballistic since the length of the wire is not much longer than the mean-free path in $\mathrm{Ni}^{20}$ The electronic structure is described using a free-electron model. The exchange splitting of the ferromagnetic band is introduced using the Stoner model via an effective exchange field, $E_{\mathrm{ex}}$. The ballistic conductance is calculated using a transfer matrix method which is outlined below.

The spin-dependent wave function of a contact with square cross section $L \times L$ is expanded in terms of the transverse eigenfunctions

$$
\Psi(x, y, z)=\sum_{l, m} \sin \left(\frac{l \pi x}{L}\right) \sin \left(\frac{m \pi y}{L}\right)\left(\begin{array}{l}
\psi_{l m}^{\uparrow}(z) \\
\psi_{l m}^{\dagger}(z)
\end{array}\right),
$$

where the spin components $\psi_{l m}^{\uparrow}(z)$ and $\psi_{l m}^{\downarrow}(z)$ obey the Schrödinger equation:

$$
\begin{aligned}
& \left\{\left[\frac{\hbar^{2}}{2 m}\left(\frac{\partial^{2}}{\partial z^{2}}-\kappa_{l m}^{2}\right)+E_{F}\right]\left(\begin{array}{ll}
1 & 0 \\
0 & 1
\end{array}\right)\right. \\
& \left.\quad-E_{e x}\left(\begin{array}{cc}
\cos \theta_{n} & e^{-i \phi_{n}} \sin \theta_{n} \\
e^{i \phi_{n}} \sin \theta_{n} & -\cos \theta_{n}
\end{array}\right)\right\}\left(\begin{array}{l}
\psi_{l m}^{\uparrow} \\
\psi_{l m}^{\dagger}
\end{array}\right)=0 .
\end{aligned}
$$

Here, $E_{F}$ is the Fermi energy, $\theta_{n}$ and $\phi_{n}$ are the azimuthal and polar angles of the magnetic moment in the $n$th layer, 
and $\kappa_{l m}$ is the transverse component of the wave vector

$$
\kappa_{l m}=\sqrt{\left(\frac{\pi l}{L}\right)^{2}+\left(\frac{\pi m}{L}\right)^{2}} .
$$

The solutions of Eq. (2) in the $n$th layer can be represented in the form of the matrix product

$$
\begin{aligned}
\left(\begin{array}{l}
\psi_{l m}^{\uparrow} \\
\psi_{l m}^{\downarrow}
\end{array}\right)= & \left(\begin{array}{cc}
\cos \frac{\theta_{n}}{2} & -e^{-i \phi_{n}} \sin \frac{\theta_{n}}{2} \\
e^{i \phi_{n}} \sin \frac{\theta_{n}}{2} & \cos \frac{\theta_{n}}{2}
\end{array}\right) \\
& \times\left(\begin{array}{l}
A_{n} e^{i k_{l m}^{\uparrow} z}+B_{n} e^{-i k_{l m}^{\uparrow} z} \\
C_{n} e^{i k_{l m}^{\downarrow} z}+D_{n} e^{-i k_{l m}^{\downarrow} z}
\end{array}\right),
\end{aligned}
$$

where

$$
k_{l m}^{\uparrow, \downarrow}=\sqrt{\frac{2 m}{\hbar^{2}}\left(E_{F} \pm E_{\mathrm{ex}}\right)-\kappa_{l m}^{2}},
$$

is the longitudinal component of the wave vector. The coefficients $A_{n}, B_{n}, C_{n}$, and $D_{n}$ are found by matching the wave function and its derivative at each layer interface. In doing so, we can write a transfer matrix equation for the amplitudes in the $N$ th layer in terms of the incident amplitudes in the first layer. Solving this equation with the boundary conditions $A_{1}=1 / \sqrt{k_{l m}^{\uparrow}}, C_{1}=0, B_{N}=D_{N}=0$ for the incoming upspin wave and $A_{1}=0, C_{1}=1 / \sqrt{k_{l m}^{\downarrow}}, B_{N}=D_{N}=0$ for the incoming down-spin wave, we find the transmission coefficient

$$
T=\sum_{\sigma l m}\left[k_{l m}^{\uparrow}\left|A_{N}\right|^{2} \boldsymbol{s}\left(k_{l m}^{\uparrow}\right)+k_{l m}^{\downarrow}\left|C_{N}\right|^{2} \boldsymbol{s}\left(k_{l m}^{\downarrow}\right)\right] .
$$

Here, the summation over $\sigma$ reflects the contributions from the incoming up-spin and down-spin waves. The function $\boldsymbol{s}(k)$ is introduced to exclude evanescent waves: $s=1$ for real $k$ and $\varsigma=0$ for imaginary $k$. The conductance is given by $G$ $=\left(e^{2} / h\right) T .^{21}$ In our calculations, we use the commonly accepted values of the Fermi energy and the exchange splitting for $\mathrm{Ni}: E_{F}=3.7 \mathrm{eV}, E_{e x}=0.5 \mathrm{eV} .{ }^{8,12}$

First, we perform a test calculation of the ballistic conductance as a function of the DW width for a nanowire consisting of ten segments of $1 \mathrm{~nm}$ width and $2 \mathrm{~nm} \times 2 \mathrm{~nm}$ cross section. The DW is assumed to have a $\tanh (\pi z / w)$ Bloch shape, where $w$ is the DW width. We find that the conductance increases rapidly from $G \approx 39 e^{2} / h$ for $w=0$ to $G=50 e^{2} / h$ for $w \rightarrow \infty$, thereby showing a positive DW resistance. This result is not unexpected, because the mixing of up- and down-spin electrons in a DW arises from a mistracking of electron spin on passing through the DW. In the ballistic regime, the narrower the DW width, the stronger the electron spin lags behind the local magnetization orientation in the DW, thereby enhancing the resistance. ${ }^{2}$ Note that the DW configuration is similar to that of a GMR magnetic trilayer: Two regions of oppositely pointing magnetizations are separated by a spacer region, i.e., by the DW. ${ }^{22}$

Figure 1(a) shows results of micromagnetic modeling for a nanojunction with soft asymmetric electrodes of dimensions $30 \mathrm{~nm} \times 24 \mathrm{~nm} \times 24 \mathrm{~nm}$ and $72 \mathrm{~nm} \times 24 \mathrm{~nm} \times 24 \mathrm{~nm}$. In these calculations, the materials parameters typical for $\mathrm{Ni}$ are used: Exchange stiffness $A=3.4 \times 10^{-7} \mathrm{erg} / \mathrm{cm}$, bulk cubic anisotropy $K_{1}=-5.7 \times 10^{4} \mathrm{erg} / \mathrm{cm}^{3}$, and saturation magnetization $M_{s}=490 \mathrm{emu} / \mathrm{cm}^{3}$. The difference in the shape Downloaded 13 Feb 2007 to 129.93.16.206. Redistribution subject
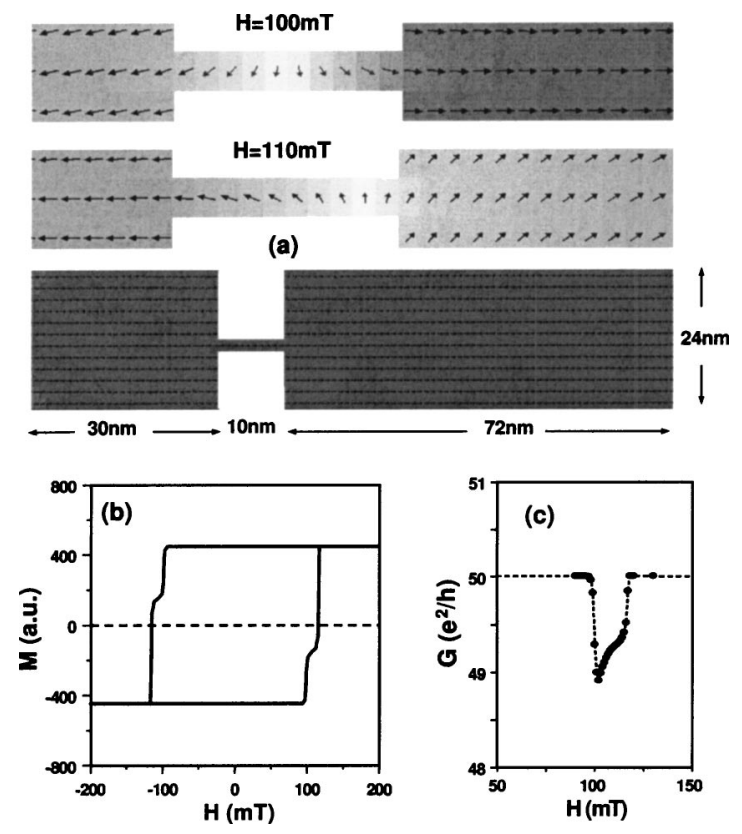

FIG. 1. Micromagnetic structure and conductance of an asymmetric $\mathrm{Ni}$ nanojunction: (a) Magnetic structure of the full-size junction at saturation (bottom panel) and of the junction's central region for $H=100 \mathrm{mT}$ and $H$ $=110 \mathrm{mT}$ (top panels), (b) hysteresis loop, and (c) conductance vs applied field.

anisotropy makes the left-hand side electrode slightly easier to switch than the right. This can be seen from the hysteresis loop shown in Fig. 1(b). As we apply a field parallel to the junction axis, the magnetization of the left-hand side electrode switches before the right, yielding an antiparallel alignment of the two electrodes within the switching region from $100 \mathrm{mT}$ to $120 \mathrm{mT}$. It is in this region that a DW is formed within the constriction [see the top panels in Fig. 1(a)]. The DW is largely confined to the constriction, ${ }^{23}$ because the shape anisotropy makes the DW much narrower than in the bulk. Indeed, assuming bulk anisotropy $K=K_{1}$ and taking into account that $w \approx 2(A /|K|)^{1 / 2}$, we obtain $w \approx 50 \mathrm{~nm}$, whereas shape anisotropy $K=\mu_{o} M_{s}^{2} / 2$ yields $w \approx 10 \mathrm{~nm}$.

Figure 1(c) shows the calculated conductance in the switching region. For a uniformly magnetized nanojunction, the conductance is $G=50 e^{2} / h$. As is seen from Fig. 1(c), the conductance drops noticeably in the switching region. The initial decrease in the conductance at $H \approx 100 \mathrm{mT}$ is due to the switching of the left-hand side electrode and the formation of the DW. Then, as the field increases, the DW moves to the right extending to the right-hand side electrode [see the middle panel in Fig. 1(a)]. The increased DW width leads to a monotonic rise in the conductance. Eventually, the righthand side electrode switches making the constriction region uniformly magnetized, and the conductance returns to its saturation value. Note that the growth of the junction conductance with the increasing magnetic field is due to the soft nature of the electrodes which allows a nonuniform magnetization to extend beyond the constriction region.

This behavior changes dramatically if one of the electrodes is magnetically hard. We consider a configuration where the left-hand side electrode is $\mathrm{Ni}$, while the right-hand side electrode is CoPt with the easy axis parallel to the axis of the wire. Both electrodes have dimensions $30 \mathrm{~nm}$ $\times 24 \mathrm{~nm} \times 24 \mathrm{~nm}$. For the CoPt, we use exchange stiffness $A=9 \times 10^{-7} \mathrm{erg} / \mathrm{cm}$, uniaxial anisotropy $K=3 \times 10^{7} \mathrm{erg} /$ to AIP license or copyright, see http://apl.aip.org/apl/copyright.jsp 

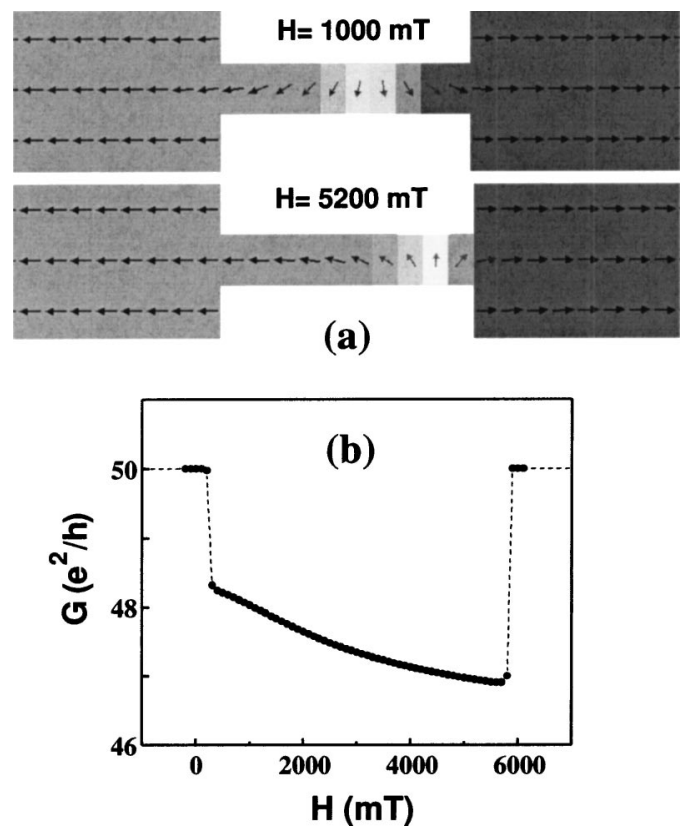

FIG. 2. (a) Magnetic structure within the constriction for a nanojunction with soft and hard electrodes for $H=1000 \mathrm{mT}$ and $H=5200 \mathrm{mT}$ and (b) conductance vs applied field.

$\mathrm{cm}^{3}$, and saturation magnetization $M_{s}=800 \mathrm{emu} / \mathrm{cm}^{3}$. We find that a DW is now localized within the constriction for a much broader range of the applied field values, namely from $500 \mathrm{mT}$ to $6000 \mathrm{mT}$. As is seen from the top panel of Fig. 2(a), at $H=1000 \mathrm{mT}$ the DW width is about the same as the one for the homogeneous junction shown in the top panel of Fig. 1(a). However, in the case of the hard right-hand side electrode, due to its very large anisotropy the increasing magnetic field (up to $6000 \mathrm{mT}$ ) is not able to push the DW out the constriction and, hence, compresses it [see the bottom panel of Fig. 2(a)]. As is evident from Fig. 2(b), this compression of the DW leads to a sizeable decrease in the conductance. Therefore, the magnetic field controls the width and the resistance of the DW localized in the nanoconstriction. We find a similar phenomenon for geometry with the easy axis of the CoPt electrode oriented perpendicular to the axis of the nanojunction if a field is applied along the easy axis.

In conclusion, we have shown that using nanoconstriction geometry with a wire of a few $\mathrm{nm}$ in cross section and about $10 \mathrm{~nm}$ in length connecting two ferromagnetic elec- trodes, it is possible to trap the DW within the constriction region. If the electrodes have significantly different magnetic anisotropies, the DW can be compressed by an applied magnetic field resulting in the enhanced DW resistance. This implies that the DW resistance can be controlled by the applied magnetic field.

This work is supported by Seagate Research, the NSF (Grant Nos. DMR-0203359 and MRSEC: DMR-0213808), and the W. M. Keck Foundation.

${ }^{1}$ A. D. Kent, J. Yu, U. Rüdiger, and S. S. P. Parkin, J. Phys.: Condens. Matter 13, R461 (2001)

${ }^{2}$ G. G. Cabrera and L. M. Falicov, Phys. Status Solidi B 61, 539 (1974).

${ }^{3}$ U. Ruediger, J. Yu, S. Zhang, A. D. Kent, and S. S. P. Parkin, Phys. Rev. Lett. 80, 5639 (1998).

${ }^{4}$ D. Ravelosona, A. Cebollada, F. Briones, C. Diaz-Paniaga, F. Hidalgo, and F. Batallan, Phys. Rev. B 59, 4322 (1999).

${ }^{5}$ U. Ebels, A. Radulescu, Y. Henry, L. Piraux, and K. Ounadjela, Phys. Rev. Lett. 84, 983 (2000).

${ }^{6}$ R. Danneau, P. Warin, J. P. Attané, I. Petej, C. Beigné, C. Fermon, O. Klein, A. Marty, F. Ott, Y. Samson, and M. Viret, Phys. Rev. Lett. 88, 157201 (2002).

${ }^{7}$ G. Tatara and H. Fukuyama, Phys. Rev. Lett. 78, 3773 (1997).

${ }^{8}$ P. M. Levy and S. Zhang, Phys. Rev. Lett. 79, 5110 (1997).

${ }^{9}$ R. P. van Gorkom, A. Brataas, and G. E. W. Bauer, Phys. Rev. Lett. 83, 4401 (1999).

${ }^{10}$ N. García, M. Muñoz, and Y.-W. Zhao, Phys. Rev. Lett. 82, 2923 (1999).

${ }^{11}$ P. Bruno, Phys. Rev. Lett. 83, 2425 (1999).

${ }^{12}$ H. Imamura, N. Kobayashi, S. Takahashi, and S. Maekawa, Phys. Rev. Lett. 84, 1003 (2000).

${ }^{13}$ L. R. Tagirov, B. P. Vodopyanov, and K. B. Efetov, Phys. Rev. B 65, 214419 (2002)

${ }^{14}$ V. K. Dugaev, J. Berakdar, and J. Barnas, Phys. Rev. B 68, 104434 (2003).

${ }^{15}$ M. Ye. Zhuravlev, E. Y. Tsymbal, S. S. Jaswal, A. V. Vedyayev, and B. Dieny, Appl. Phys. Lett. 83, 3534 (2003).

${ }^{16}$ J. Kudrnovsky, V. Drchal, C. Blaas, P. Weinberger, I. Turek, and P. Bruno, Phys. Rev. B 62, 15084 (2000).

${ }^{17}$ B. Yu. Yavorsky, I. Mertig, A. Ya. Perlov, A. N. Yaresko, and V. N. Antonov, Phys. Rev. B 66, 174422 (2002).

${ }^{18}$ A. K. Solanki, R. F. Sairyanov, E.Y. Tsymbal, and S. S. Jaswal, J. Magn. Magn. Mater. 272-276, 1730 (2004).

${ }^{19}$ M. J. Donahue and D. G. Porter, oOMmF User's Guide version 1.0 (NIST, Gaithersburg, MD, 1999) Interagency Report NISTIR 6376; http:// math.nist.gov/oommf/

${ }^{20}$ B. A. Gurney, V. S. Speriosu, J. P. Nozieres, H. Lefakis, D. R. Wilhoit, and O. U. Need, Phys. Rev. Lett. 71, 4023 (1993).

${ }^{21}$ R. Landauer, IBM J. Res. Dev. 1, 223 (1957).

${ }^{22}$ E. Y. Tsymbal and D. G. Pettifor, in Solid State Physics, edited by H. Ehrenreich and F. Spaepen (Academic, San Diego, 2001), Vol. 56, pp. 113-237.

${ }^{23}$ R. Skomski, J. Phys.: Condens. Matter 15, R841 (2003). 